\title{
Téoros
}

Revue de recherche en tourisme

\section{Le tourisme international au Maroc}

\section{Jean Stafford et Bruno Sarrasin}

Volume 19, numéro 3, automne 2000

URI : https://id.erudit.org/iderudit/1071745ar

DOI : https://doi.org/10.7202/1071745ar

Aller au sommaire du numéro

Éditeur(s)

Université du Québec à Montréal

ISSN

0712-8657 (imprimé)

1923-2705 (numérique)

Découvrir la revue

Citer cette note

Stafford, J. \& Sarrasin, B. (2000). Le tourisme international au Maroc. Téoros, 19(3), 51-54. https://doi.org/10.7202/1071745ar d'utilisation que vous pouvez consulter en ligne.

https://apropos.erudit.org/fr/usagers/politique-dutilisation/ 


\section{LE TOURISME INTERNATIONAL AU MAROC}

\section{Jean Stafford et Bruno Sarrasin}

Cette chronique porte sur la conjoncture touristique. Elle propose un portrait simplifié de la situation du tourisme à partir des données disponibles. Les notions usuelles de l'analyse prévisionnelle (les tendances passées, présentes et futures) sont utilisées et des prévisions pour l'horizon 2003 sont proposées.

Le Maroc est un pays touristique privilégié par sa position géographique. Sa vocation touristique naturelle s'appuie notamment sur son appartenance au bassin méditerranéen qui regroupe les principaux pays émetteurs de touristes que constitue l'Union européenne. Cette situation géographique unique, sur l'Atlantique et la Méditerranée, offre presque toute l'année un climat tempéré sur une grande partie du territoire marocain.

On peut résumer 1'offre touristique marocaine autour de trois « ensembles » :

- les littoraux atlantique et méditerranéen dont Agadir est la destination la mieux connue des touristes internationaux ;

- les massifs montagneux du Rif et de l'Atlas jusqu'au Sahara marocain, dont Ouarzazate constitue un passage quasi obligé ;

- un passé culturel très riche, façonné par une longue histoire qui chevauche les civilisations romaine et islamique, dont l'offre touristique s'articule autour des quatre cités impériales : Fès, Meknès, Marrakech et Rabat.

Conscientes de l'importance de ce capital touristique, encouragées par les recommandations de la Banque mondiale depuis les années 1960 et par le succès touristi- que remporté par leur voisin du Nord l'Espagne -, les autorités marocaines se sont résolument engagées sur la voie du tourisme en 1965 (Stafford, Bélanger et Sarrasin, 1996). Ce choix s'appuie sur le potentiel géographique et climatique que nous avons évoqué, mais aussi sur des raisons d'ordre socio-économique et politique comme nous le montrerons brièvement dans cet article. Il faut d'abord bien saisir l'importance que représente ce secteur pour la croissance de l'économie marocaine.

Le secteur touristique représente plus de $15 \%$ des exportations directes du Maroc et arrive au second rang en importance, après les transferts des Marocains résidant à l'étranger ${ }^{1}$. En 1999, près de deux millions de touristes étrangers ont visité le pays, ce qui représente pour ce secteur une contribution directe de $4,7 \%$ et une contribution indirecte de $3 \%$ sur la croissance globale de l'économie (produit national brut). L'importance de ce secteur sur l'économie demeure cependant bien en deçà du ratio de ses principaux concurrents telles la Tunisie $(13,9 \%)$, la Grèce $(18,3 \%)$ et la Turquie $(16,5 \%)$. Ces données révèlent en fait que l'économie du Maroc est moins « dépendante » du secteur touristique que ces pays. Son importance « sociale »peut se mesurer par les 330000 emplois directs
(4,5\% du total) et les 210000 emplois induits ( $3 \%$ du total) que l'industrie touristique marocaine génère, parmi lesquels les emplois liés au commerce de détail et au secteur de la construction sont les plus importants.

Le poids relativement faible du tourisme dans l'économie marocaine pourrait laisser



Porteurs d'eau à Marrakech 
présager un potentiel sous-exploité. Ce secteur est pourtant relativement stagnant depuis près de dix ans, résultat d'un code d'investissement touristique particulièrement libéral au cours des années 1970 et 1980 qui a mené à un déséquilibre de l'offre par rapport à la demande, particulièrement en ce qui concerne la qualité et la diversité des produits offerts. De 1988 à 1997 , le nombre de touristes internationaux (Algériens exclus) a augmenté en moyenne de $1,4 \%$ par année en comparaison à $8,3 \%$ pour l'ensemble du Moyen-Orient et à $9 \%$ dans le monde. En 1998, des 90000 lits que constituait l'offre hôtelière marocaine, seuls 60000 pouvaient répondre à la demande internationale.

Bien que le secteur du tourisme ne réponde pas exclusivement à la demande internationale, celle-ci constitue un atout particulier pour un pays comme le Maroc dont la dette est essentiellement financée par l'étranger. Comme tout secteur d'exportation, le tourisme international est pourvoyeur de devises (ce qui n'est pas le cas du tourisme national), contribuant au paiement des importations et au service de la dette ${ }^{2}$. Pour mieux saisir la place du tourisme au Maroc, nous proposons dans cet article d'analyser son évolution au cours des dix dernières années (1988 à 1999 ) et nous faisons des prévisions jusqu'en 2003.

Le tableau 1 présente trois périodes de quatre années, chacune marquée par des

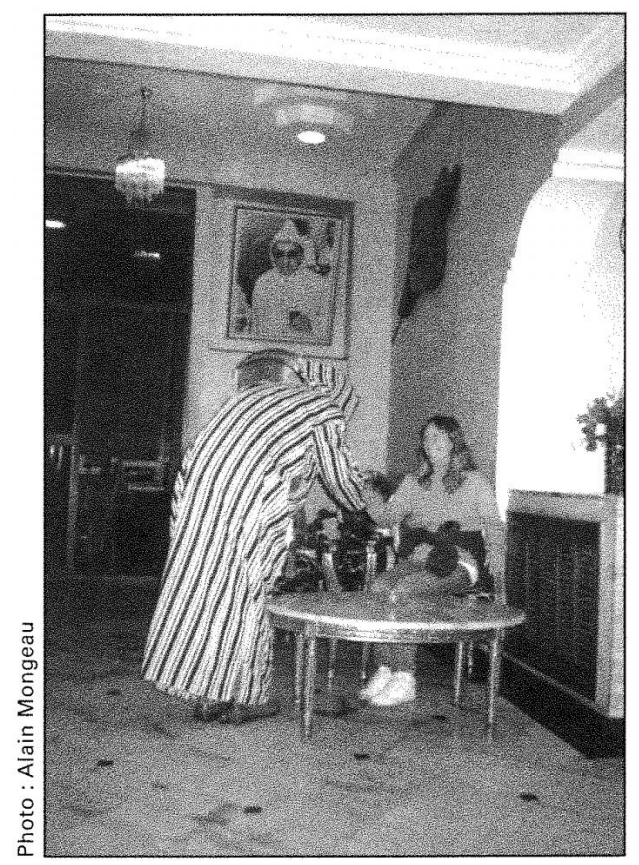

Hall d'entrée de l'Hôtel de Paris à Casablanca

\begin{tabular}{|c|c|c|}
\hline \multicolumn{3}{|c|}{ TABLEAU 1 } \\
$\begin{array}{c}\text { Les taux d'accroissement ammuel moyen (moyemue géométrique) des nuitées des touristes } \\
\text { étrangers au Maroc, par segments de quatre années (1988 à 1999) }\end{array}$ \\
\hline Années & Nuitées & Taux d'accroissement annuel moven \\
\hline 1988 & 8782262 & $-15,5 \%$ \\
\hline 1989 & 8876131 & \\
\hline 1990 & 8287285 & \\
\hline 1991 & 5303918 & \\
\hline 1992 & 8816909 & \\
\hline 1993 & 9309657 & \\
\hline 1994 & 9325546 & \\
\hline 1995 & 7775392 & \\
\hline 1996 & 7886191 & \\
\hline 1997 & 8645002 & \\
\hline 1998 & 9701359 & \\
\hline 1999 & 10845778 & \\
\hline
\end{tabular}

Source : ministère du Tourisme, Royaume du Maroc

\section{GRAPHIOUE 1}

Les parts de marché des nuitées de la france et de IAllemagne au Maroc. par annee, de 1987 a 1999






\section{GRAPHIQUE 2}

\section{La prévision des nuitées au Maroe de 1987 a 2003 selon deux hypotèses de développement}

\section{NUITÉES}

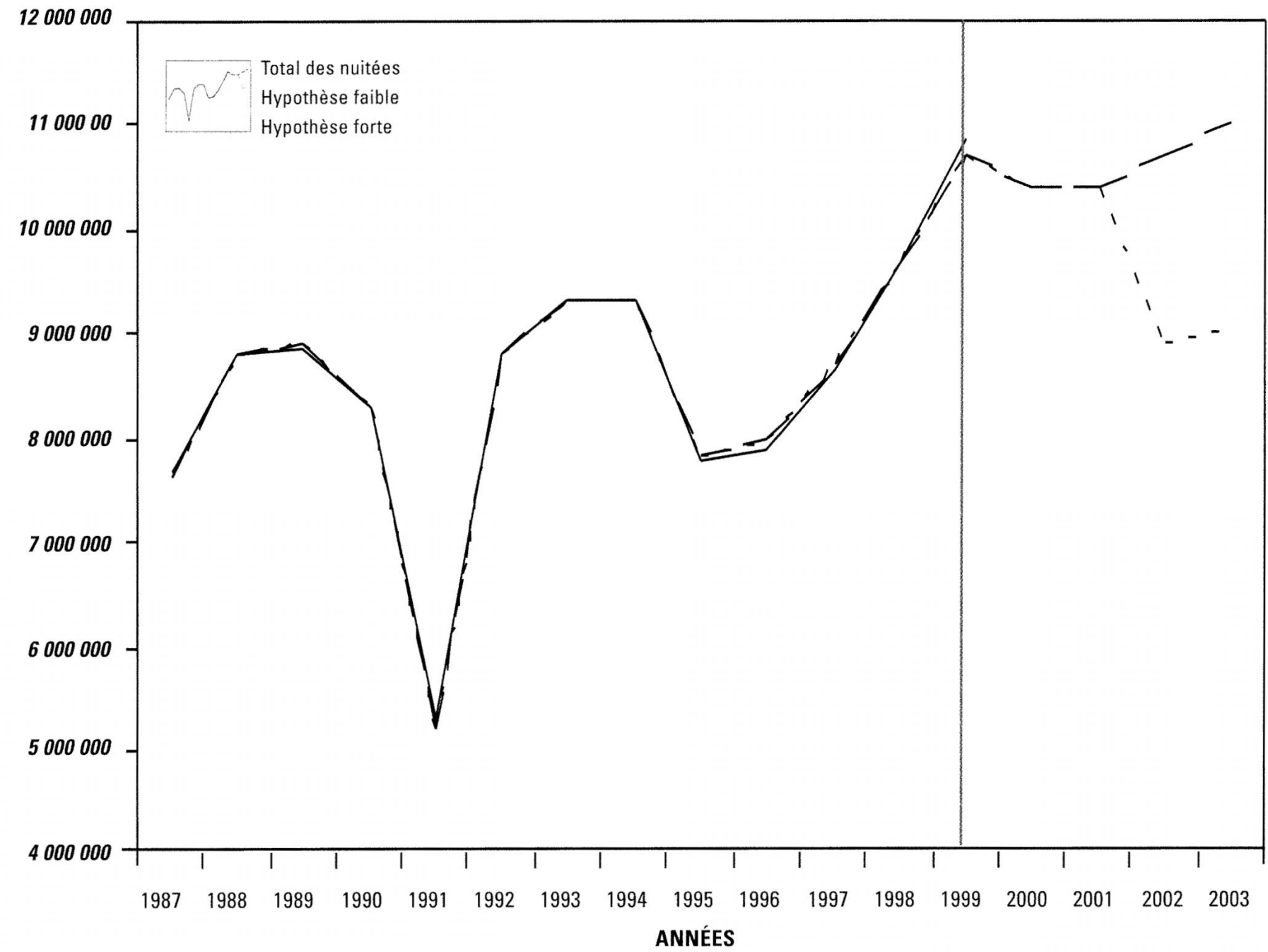

conjonctures particulières que nous aborderons brièvement. On remarque d'abord que les nuitées de touristes étrangers ont chuté en moyenne de $-15,5 \%$ par année de 1988 à 1991. Il est important de souligner que cette baisse est amplifiée par le début du segment (1988) qui représente l'achèvement d'un cycle de croissance de l'économie mondiale et la fin du segment (1991) qui est marquée par une crise politique (Guerre du Golfe) et économique (crise du pétrole et récession) touchant l'ensemble des pays émetteurs de touristes. Cette crise du début des années 1990 a particulièrement marqué les pays arabes à tradition touristique (Égypte, Tunisie, Maroc) à cause de la perception de la
Guerre du Golfe par les Occidentaux. De 1990 à 1991 seulement, les nuitées de touristes internationaux au Maroc ont chuté de $-36 \%$.

Les deux autres segments du tableau 1 suivent, à peu de choses près, les conjonctures internationales. D'abord la reprise en 1992 des nuitées de touristes étrangers freinées un an plus tôt. L'année 1995 témoigne une baisse marquée par rapport à l'année précédente $(-16,6 \%)$ et conduit la moyenne du segment à une baisse annuelle de -4,1\%. Les années 1995 et 1996 ont pu être particulièrement affectées par l'intensification de la guerre civile en Algérie. Cette hypothèse s'appuie sur l'effet qu'ont pu avoir les médias sur les clientèles occidentales dans le traitement du conflit. Les deux principaux marchés touristiques du Maroc, la France et l'Allemagne, ont suivi une évolution différente (souvent inverse) au cours de la période étudiée (graphique 1). La relative stagnation des nuitées des Français de 1992 à 1995 (qui représentent à eux seuls plus de $50 \%$ du marché) a contribué à la baisse moyenne des nuitées pour cette période (tableau 1).

Or, la croissance reprend de 1997 à 1999 , correspondant à l'évolution des conjonctures économiques européennes, françaises en particulier. Pour l'ensemble de la période (1988 à 1999), les nuitées de touristes 
étrangers au Maroc ont augmenté de 1,9\% par année en moyenne, témoignant d'une faible hausse, sinon d'une stagnation de la demande depuis dix ans.

Nous prévoyons deux hypothèses d'évolution des nuitées de touristes étrangers au Maroc pour l'horizon 2003 (graphique 2). Ces hypothèses reposent sur l'évolution anticipée d'un cycle économique correspondant à celui des principaux marchés touristiques du Maroc, en particulier la France et l'Allemagne. L'hypothèse faible s'appuie sur l'apport d'un cycle positif en $2000(+9 \%)$ et $2001(+7 \%)$, pour ensuite décliner à $-12 \%$ en 2002 et en 2003. Ces pourcentages sont des hypothèses d'évolution qui correspondent à un ralentissement marqué de l'économie des principales clientèles touristiques marocaines.

L'hypothèse forte correspond à un cycle conjoncturel positif de $9 \%$ en 2000 , de $7 \%$ en 2001 , de $9 \%$ en 2002 et de $11 \%$ en 2003. Il est important de souligner que l'analyse du cycle de 1988 à 1999 montre un apport positif de $4 \%$ en 1998 et de $15 \%$ en 1999 . Poser l'hypothèse d'un cycle positif de $9 \%$ en 2000 et de $7 \%$ en 2001 correspond donc à un ralentissement de l'économie pour ces années, bien que l'effet sur les nuitées au Maroc demeure positif.

\section{CONCLUSION}

L'industrie touristique du Maroc est en forte croissance pour la dernière période étudiée (1996-1999). Quant à l'horizon 20002003, deux hypothèses d'évolution sont proposées, mais selon l'information que nous possédons actuellement (novembre 2000) la deuxième hypothèse, celle d'une croissance forte, semble la plus probable.

Pour l'ensemble de la période étudiée 1988-1999 (et cela est valable pour les vingt dernières années), la France représente le marché touristique le plus important ; l'évolution de ce marché spécifique détermine fortement la situation de l'industrie touristique marocaine ${ }^{3}$. Cette forte dépendance est à la fois une force et une faiblesse : bien que le Maroc puisse compter sur une clientèle fidèle et nombreuse, il est dangereux de dépendre d'une seule clientèle. Le Maroc devrait donc se donner comme objectif à moyen terme d'augmenter graduellement les clientèles du bassin méditerranéen et, comme objectif à long terme, d'essayer d'attirer d'autres clientèles internationales.

Jean Stafford est professeur et Bruno Sarrasin est chargé de cours, tous deux au Département d'études urbaines et touristiques de l'UQAM.

\section{NOTES}

1 Les renseignements économiques, sauf indication contraire, proviennent de la Banque mondiale, particulièrement de la description du projet Morocco - Tourism Development approuvé le 8 juin 2000 .

(www.worldbank.org)

2 Pour avoir un aperçu du débat concernant le rôle des exportations dans l'économie des pays du Sud, voir Sarrasin (1999).

3 Une analyse de régression multiple (réalisée par les auteurs), incluant les principaux marchés touristiques du Maroc comme variables indépendantes et les nuitées touristiques comme variable dépendante, montre que la France seule pourrait être une variable suffisante (pour ce qui est de la variance expliquée) pour prévoir l'évolution du tourisme au Maroc au cours des cinq prochaines années.

\section{BIBLIOGRAPHIE}

Sarrasin, Bruno (1999), Ajustement et lutte contre la pauvreté en Afrique subsaharienne. La Banque mondiale face à la critique, Paris, L'Harmattan.

Stafford, Jean, Charles Bélanger, et Bruno Sarrasin (1996), Tourisme et développement au Maroc, Montréal, L'Harmattan.



Centre-ville de Casablanca 